NASA/TM—2006-214390

\title{
Responding to Mechanical Antigravity
}

Marc G. Millis

Glenn Research Center, Cleveland, Ohio

Nicholas E. Thomas

University of Miami, Miami, Florida 


\section{NASA STI Program . . . in Profile}

Since its founding, NASA has been dedicated to the advancement of aeronautics and space science. The NASA Scientific and Technical Information (STI) program plays a key part in helping NASA maintain this important role.

The NASA STI Program operates under the auspices of the Agency Chief Information Officer. It collects, organizes, provides for archiving, and disseminates NASA's STI. The NASA STI program provides access to the NASA Aeronautics and Space Database and its public interface, the NASA Technical Reports Server, thus providing one of the largest collections of aeronautical and space science STI in the world. Results are published in both non-NASA channels and by NASA in the NASA STI Report Series, which includes the following report types:

- TECHNICAL PUBLICATION. Reports of completed research or a major significant phase of research that present the results of NASA programs and include extensive data or theoretical analysis. Includes compilations of significant scientific and technical data and information deemed to be of continuing reference value. NASA counterpart of peer-reviewed formal professional papers but has less stringent limitations on manuscript length and extent of graphic presentations.

- TECHNICAL MEMORANDUM. Scientific and technical findings that are preliminary or of specialized interest, e.g., quick release reports, working papers, and bibliographies that contain minimal annotation. Does not contain extensive analysis.

- CONTRACTOR REPORT. Scientific and technical findings by NASA-sponsored contractors and grantees.
- CONFERENCE PUBLICATION. Collected papers from scientific and technical conferences, symposia, seminars, or other meetings sponsored or cosponsored by NASA.

- SPECIAL PUBLICATION. Scientific, technical, or historical information from NASA programs, projects, and missions, often concerned with subjects having substantial public interest.

- TECHNICAL TRANSLATION. Englishlanguage translations of foreign scientific and technical material pertinent to NASA's mission.

Specialized services also include creating custom thesauri, building customized databases, organizing and publishing research results.

For more information about the NASA STI program, see the following:

- Access the NASA STI program home page at http://www.sti.nasa.gov

- E-mail your question via the Internet to help@sti.nasa.gov

- Fax your question to the NASA STI Help Desk at 301-621-0134

- Telephone the NASA STI Help Desk at 301-621-0390

- Write to:

NASA STI Help Desk

NASA Center for AeroSpace Information 7115 Standard Drive Hanover, MD 21076-1320 
NASA/TM-2006-214390

\section{Responding to Mechanical Antigravity}

Marc G. Millis

Glenn Research Center, Cleveland, Ohio

Nicholas E. Thomas

University of Miami, Miami, Florida

Prepared for the

42nd Joint Propulsion Conference and Exhibit cosponsored by the AIAA, ASME, SAE, and ASEE

Sacramento, California, July 9-12, 2006

National Aeronautics and

Space Administration

Glenn Research Center

Cleveland, Ohio 44135 
Trade names and trademarks are used in this report for identification only. Their usage does not constitute an official endorsement, either expressed or implied, by the National Aeronautics and Space Administration.

This work was sponsored by the Fundamental Aeronautics Program at the NASA Glenn Research Center.

Level of Review: This material has been technically reviewed by technical management.

Available from

NASA Center for Aerospace Information 7115 Standard Drive

Hanover, MD 21076-1320
National Technical Information Service 5285 Port Royal Road Springfield, VA 22161 


\title{
Responding to Mechanical Antigravity
}

\author{
Marc G. Millis \\ National Aeronautics and Space Administration \\ Glenn Research Center \\ Cleveland, Ohio 44135 \\ Nicholas E. Thomas \\ University of Miami \\ Miami, Florida 33124
}

\begin{abstract}
Based on the experiences of the NASA Breakthrough Propulsion Physics Project, suggestions are offered for constructively responding to proposals that purport breakthrough propulsion using mechanical devices. Because of the relatively large number of unsolicited submissions received (about 1 per workday) and because many of these involve similar concepts, this report is offered to help the would-be submitters make genuine progress as well as to help reviewers respond to such submissions. Devices that use oscillating masses or gyroscope falsely appear to create net thrust through differential friction or by misinterpreting torques as linear forces. To cover both the possibility of an errant claim and a genuine discovery, reviews should require that submitters meet minimal thresholds of proof before engaging in further correspondence; such as achieving sustained deflection of a level-platform pendulum in the case of mechanical thrusters.
\end{abstract}

\section{Nomenclature}

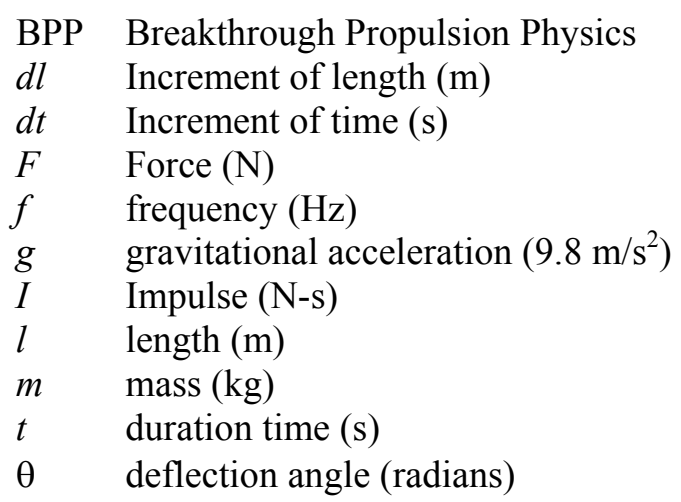

\section{Introduction}

From 1996 to 2002, NASA sponsored research into Breakthrough Propulsion Physics (BPP) that produced 14 peer-reviewed articles (ref. 1). A summary of these findings and research by others indicates that this is a nascent topic encompassing many differing approaches and challenges (ref. 2). Because of the provocative nature of seeking breakthroughs, this work receives much media attention, being cited in Newsweek (ref. 3), Wired (ref. 4), the cover of Popular Science (ref. 5), New York Times (ref. 6), The Boston Globe (ref. 7), and in the books Centauri Dreams (ref. 8), and in I'm Working On That (ref. 9); as just some prominent examples. 
As a consequence of the media exposure, the NASA BPP Project became overwhelmed with submissions from amateur enthusiasts wishing to contribute their ideas (ref. 7). To quantify this situation, statistics were compiled during 2000 through 2001 on all unsolicited correspondence sent into the NASA Project. The findings are presented in table I. Roughly a third of the submissions were from amateurs requesting reviews or other support for their ideas.

TABLE I.-UNSOLICITED CORRESPONDENCE STATISTICS FROM THE NASA BREAKTHROUGH PROPULSION PHYSICS PROJECT

\begin{tabular}{|c|c|}
\hline & Percent \\
\hline 1000 Unsolicited submissions per year (2000-2001) & 100 \\
\hline $\begin{array}{l}\text { Professional researchers: } \\
\text { - News of recent peer-reviewed publications } \\
\text { - Inquiries about future research solicitations } \\
\text { - Employment inquires }\end{array}$ & 32 \\
\hline $\begin{array}{l}\text { Amateur researchers requesting feedback } \\
\text { - "Here is my breakthrough..." } \\
\text { - "Please evaluate my idea..." } \\
\text { - "Please help me advance my idea..." } \\
\text { - (About a third of amateur requests, } 9 \% \text { of all submissions, display paranoia or delusions } \\
\text { of grandeur) }\end{array}$ & 31 \\
\hline $\begin{array}{l}\text { Public inquiries: } \\
\text { - "Please tell me more about..." } \\
\text { - "What is your assessment of...?" } \\
\text { - "Please help me with my homework." }\end{array}$ & 30 \\
\hline Invitations to conferences/workshops & 4 \\
\hline Press interview requests & 2 \\
\hline Public speaking requests & 1 \\
\hline
\end{tabular}

Given that an objective review and response can take roughly 3 days to prepare and considering the rate of incoming submissions, it is estimated that a fulltime staff of 3 to 4 researchers would be needed just to respond to the amateur requests. In addition to the technical issues, amateur submissions often present the additional challenge of non-professional, emotionally-charged correspondence. Even though reviews were occasionally conducted early in the BPP Project, eventually the Project had to adopt the policy of not reviewing any unsolicited submissions.

Other organizations also face the challenge of receiving amateur submissions, but there are no reference-able documents to help guide reviewers on how best to respond. As a service to other reviewers and would-be submitters, the lessons from the BPP Project are summarized here.

The quintessential example chosen for illustrating this situation is the common proposal to use oscillating masses or gyroscopes to produce net thrust. Known to violate conservation of momentum when interpreted as a breakthrough claim, such ideas are often summarily dismissed - a response that does little to educate or dissuade the submitters. To offer a more effective response, explanations are offered next on why these devices appear to be breakthroughs, how they operate from the perspective of established physics, and what the submitters can do to provide more convincing evidence of how the device really operates.

In addition, suggestions are offered on how to phrase the response to encourage the submitters to become more rigorous with their investigations so that they can learn from their experiences. 


\section{Oscillation Thrusters}

The oscillation thruster, also describable as a sticktion drive, internal drive, or slip-stick drive, is a commonly suggested device that uses the motion of internal masses to create net thrust. One of the most famous oscillation thrusters is the 1959 "Dean Drive" described in Patent 2,886,976 (ref. 10). A more recent and simple example is shown in figure 1 (ref. 11). Further still, figure 2 displays an example that uses rotating masses (ref. 12). Although there are many versions, all oscillation thrusters have the following common components:

- Chassis to support a system of masses

- Conveyor that moves the masses through an asymmetric cycle

- Power source for the conveyor

A crucial feature is that the internal masses go through a cyclic motion where the motion in one direction is quicker than in the other. The result is that the whole device moves in surges across the ground, giving the appearance that a net thrust is being produced without expelling a reaction mass or having a direct driving connection to the ground.

Because it would constitute a breakthrough to be able to move a vehicle without using a reaction mass (ref. 2), these devices appear to be breakthroughs. Regrettably, such devices are not breakthroughs, since they still require a connection to the ground to create net motion. The ground is the reaction mass and the frictional connection to the ground is a necessary component to its operation.

More specifically, it is the difference between the static fiction (sometimes called sticktion) and the dynamic friction between the device and the ground that is required for their operation. Static friction, the amount of friction encountered when contacting surfaces are not moving relative to one another, is typically greater than the dynamic friction between the same materials. Dynamic friction is the amount of friction when the contacting surfaces are moving relative to one another.

Recall that the device's internal masses move fast in one direction and slow in the other. When the masses move quickly, the device has enough reaction force to overcome the static friction between itself and the ground, and the device slides. When the internal masses return slowly in the other direction, the reaction forces are not enough to overcome the static friction and the device stays in its place. The net effect is that such slip-stick motion causes the device to scoot across the floor.

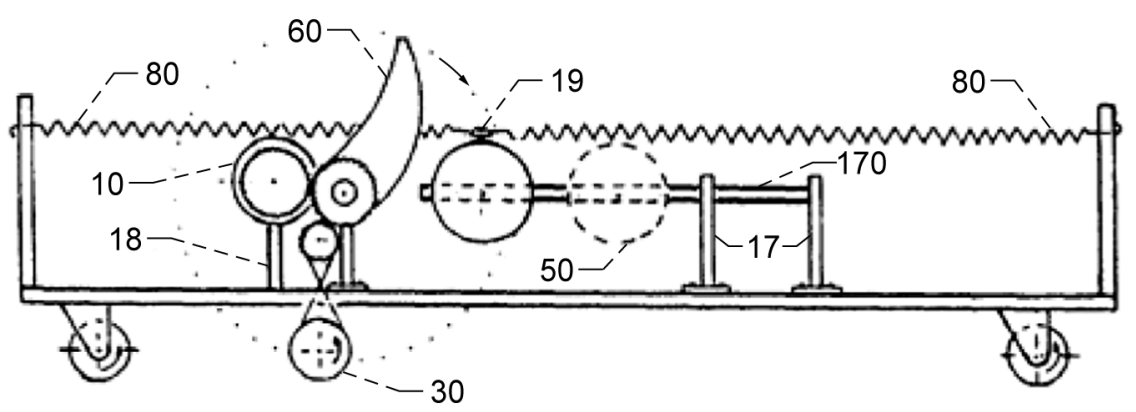

Figure 1.-Linear oscillation thruster. This is a typical example of an oscillation thruster, specifically from Patent 5,685,196 from Richard Foster (ref. 11). As the cam (60) rotates, a mass (50) moves slowly in one direction and is allowed to return quickly in the other. The reaction force from one part of this cycle is sufficient to overcome static friction, while the reaction force is insufficient in the other part of the cycle. This leads to one-directional motion, giving the illusion of net thrust. 


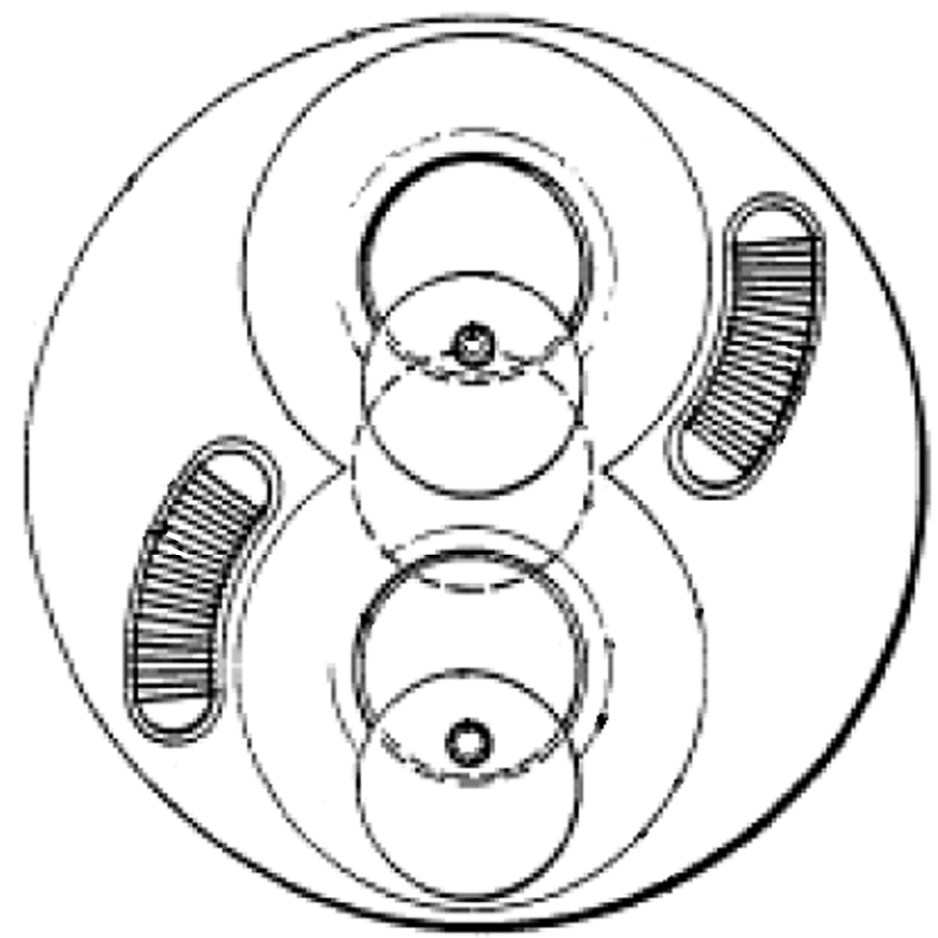

Figure 2.-Rotational oscillation thruster. This is a typical example of an oscillation thruster that uses rotating masses with convolutions of cycles. The version shown here is from Patent 4,631,971 of Brandson R. Thornson (ref. 12).

If the device could be placed into orbit to follow a freefall trajectory, absent of any connection with external masses, then the center of mass of the entire system would follow the freefall trajectory without deviation, while the device's external frame and its internal masses would just oscillate with respect to one-another. Similarly, if the device were dropped with its thrusting direction pointing either up or down, the rate of fall of the center of mass of the system would be identical regardless if the device was on or off.

To illustrate stick-slip operation, figure 3 offers an analogy. The right side of the figure represents half of a cycle where the device does not move, while the left side represents half of the cycle where the device does move. Even though the total impulse $(I=F \times t)$, as represented by the area of the rectangle, is equal in both phases of the cycle, the force, $F$, represented by the width of the rectangle, and its duration, $t$, represented by height of the rectangle, can vary. When they do, they vary reciprocally so that their product remains constant. (Note that this is only a conceptual illustration whereas a more rigorous analysis would require integrating a variable force over time.) With the impulse the same in both halfcycles, the reaction force is less on the right-hand side than on the left. When compared to the critical static friction force, $F_{c}$, which is the amount of force that would have to be overcome to set the device in motion, it is clear that the reaction force for the right half-cycle is less than this critical force, while on the left it is greater. This means that the device would move during the left hand half of the cycle, but not during the right-hand.

More rigorous analyses of a given device can take on a variety of forms, depending on the device in question. Options include using the impulse representation $\left(\int F \bullet d t\right)$ or the work representation $\left(\int F \bullet d l\right)$ over all the phases of the device's cycle. Also, depending on the device, the number of phases could vary. In 


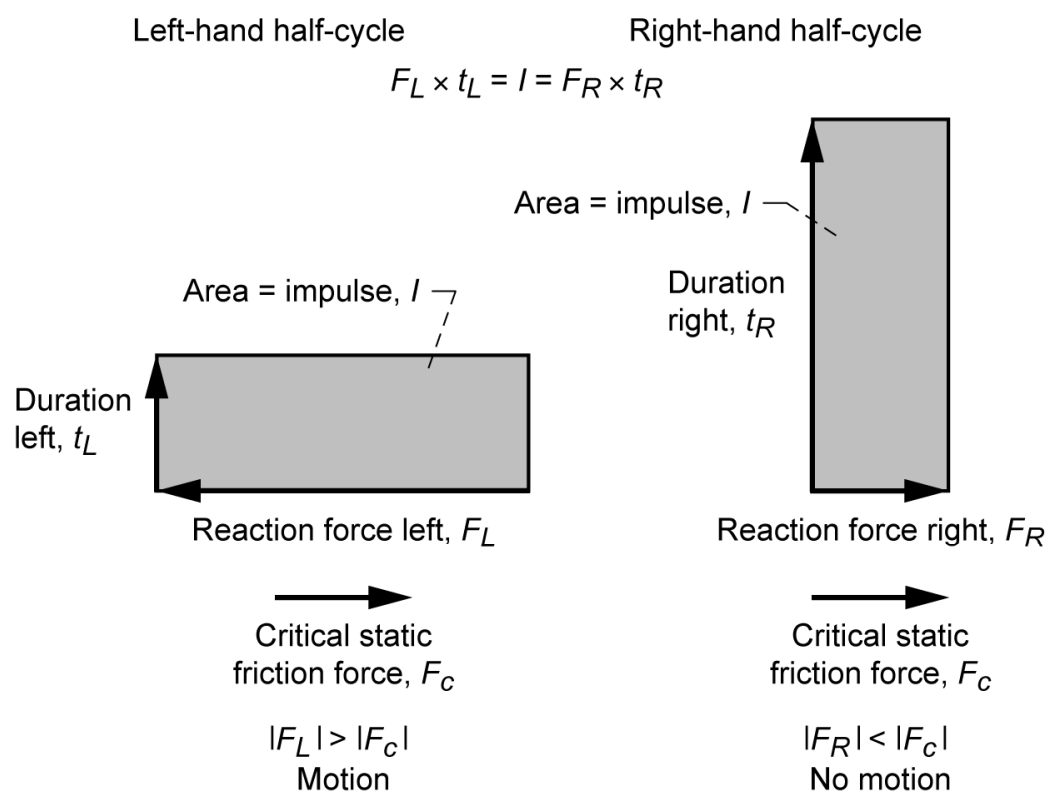

Figure 3.-Equal impulses - different forces. This is a conceptual representation of the $F \times t$ impulse of each half of a generic mechanical oscillator. Although the impulse (area of rectangle) is equal in both halves of the cycle, the force on the right part of the cycle is insufficient to overcome the critical friction, while the force is sufficient on the left. The device would move during the left half-cycle and remain stuck to the floor on the right half-cycle.

figure 3 there are only two phases, but for the Foster device in figure 1 for example, at least three phases would need to be assessed: (1) When the cam (part 60) is displacing the mass (part 50), (2) When the mass returns under the restoring force of spring (part 80), and (3) When the Mass comes to a stop back to its initial position.

Since such analyses are time consuming are not likely to be understood by many amateurs, it is more effective to suggest that the submitter produce rigorous experimental proof. The challenge is to offer an easy-to-construct test that minimizes the chance for false-positive results. A fitting test is to place the device on a level pendulum stand, as illustrated in figure 4, and compare the deflection between the on and off conditions of the device. A sustained net deflection of the pendulum is indicative of genuine thrust. Alternatively, if the pendulum oscillates around its null position, which is the expected finding, then the device is not creating net thrust.

To avoid possible spurious effects, it is advised to have the device and its power supply self-contained on the pendulum, and to have the tallest pendulum possible (long $l$ in fig. 4). The reason for containing the power supply with the device is to avoid having the power cords interfere with the free motion of the pendulum. The reasons for having a tall pendulum is to make its lateral motion ( $d$ in fig. 4$)$ more pronounced for a given lateral force, $F$, and to reduce its natural oscillation frequency to be much less than the oscillation frequency of the device. Spurious results are possible if the oscillation frequency of the device and the pendulum are similar. For reference, equations (1) and (2) present common equations for using pendulums to measure lateral thrusting (valid for small deflections, approximating $\operatorname{sine} \theta \approx \theta$ ) (ref. 13). 


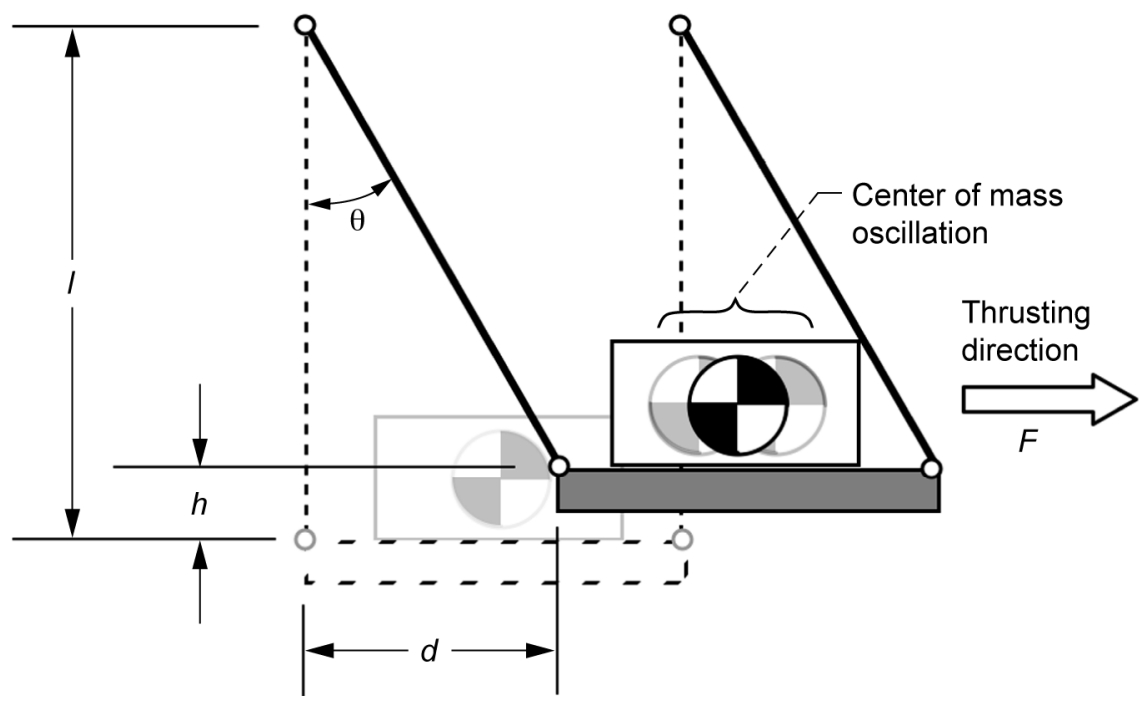

Figure 4.-Basics of a level pendulum test. A pendulum is a simple tool for measuring lateral force and a level pendulum keeps the test platform from tilting during operation. If a thrusting device can sustain a deflection of a level pendulum, then there is strong evidence toward the claim of net thrust. It is anticipated, however, that mechanical devices will instead oscillate the pendulum back and forth, with the average position being zero deflection.

Lateral Force as a Function of Deflection

$$
F=m g \tan \theta
$$

Natural Frequency of a Pendulum

$$
f=\frac{1}{2 \pi} \sqrt{\frac{g}{l}}
$$

Where

$F \quad$ lateral force acting to deflect the pendulum (N)

$f$ pendulum frequency $(\mathrm{Hz})$

$g$ gravitational acceleration $\left(9.8 \mathrm{~m} / \mathrm{s}^{2}\right)$

$l \quad$ length of the pendulum (m) (fig. 4)

$m$ mass at the lower end of the pendulum (other masses taken as negligible) $(\mathrm{kg})$

$\theta$ deflection angle (radians) (fig. 4)

The reason that a level pendulum is recommended instead of a simple pendulum is to avoid the misleading effects from tilting of the base, as illustrated in figure 5. Similarly, the reason that an air track is not recommended is because of the misleading effects from the tilting of its base, as illustrated in figure 6 . When the internal masses of the device shift off center, the base can tilt. In the case of the pendulum, this has the effect of inducing an apparent deflection of the pendulum. In the case of an air track this has the effect of deflecting more air away from the dipped end, thereby thrusting the device from the reaction to the asymmetric airflow.

To keep an open, yet rigorous, mind to the possibility that there has been some overlooked physical phenomena, it would be necessary that any future proposals on these types of devices pass a pendulum test and provide rigorous supporting data that addresses all possible false-positive conclusions. Although a "jerk" effect (time rate change of acceleration) (ref. 14) has sometimes been mentioned as a theoretical 


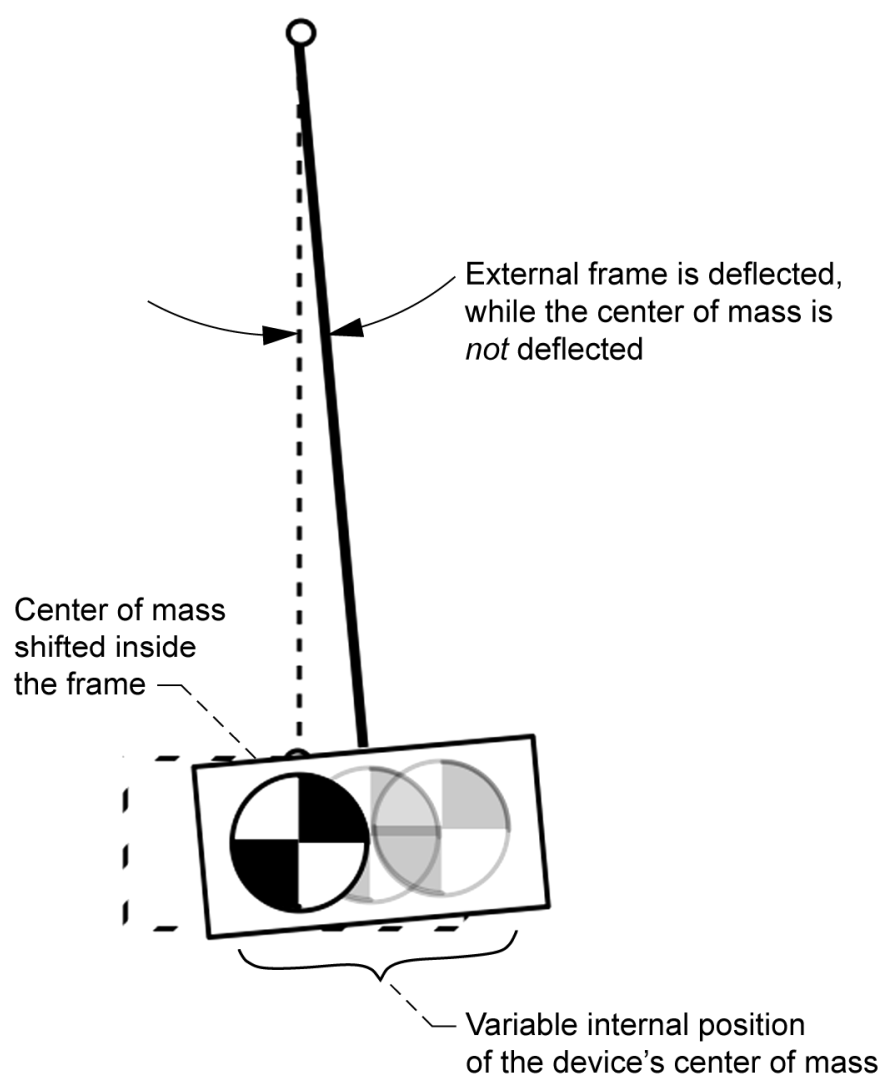

Figure 5.-Pitfall of simple pendulum. With a simple pendulum, a shift in the center of mass of the device (from the motion of its internal masses), gives the false appearance of a deflection when in fact the center of mass of the system is still directly underneath the support.

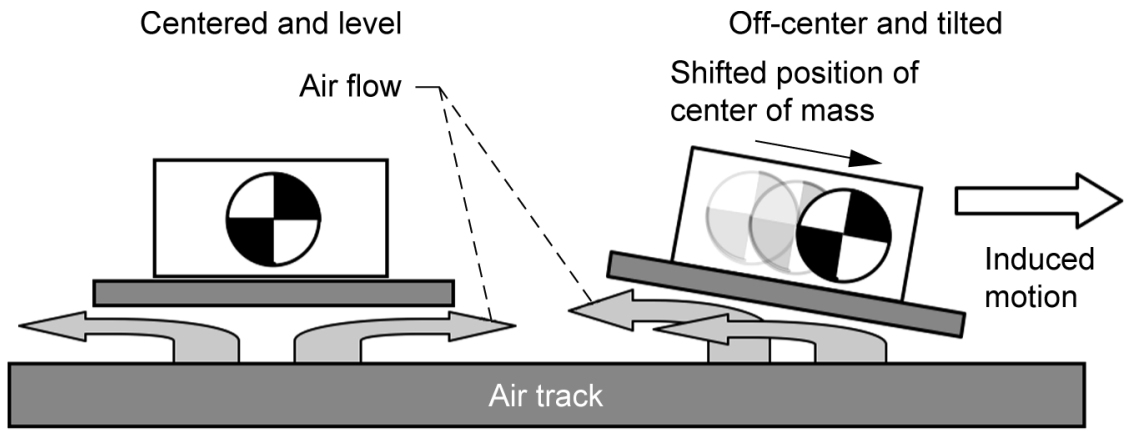

Figure 6.-Pitfalls of air track test. In addition to the fact that air tracks possess some friction, they are not suitable as a testing platform due to the potential for induced tilting. If the center of mass of the device shifts forward for example, the leading edge of the air platform can tilt downward, creating a preferred channel for the airflow, which in turn, creates a propulsive force on the platform. 
approach to understand such devices, no experiments nor physical evidence that substantiate such a jerk effect have been reported. If successful net-thrust tests are ever produced and if a genuine new effect is found, then science will have to be revised because it would then appear as if such devices were violating conservation of momentum.

One venue through which to potentially address conservation of momentum in the absence of an obvious reaction mass is to invoke a literal interpretation of Mach's principle. Mach's principle deals with the relationship between an inertial frame and the surrounding mass of the universe (ref. 15). A literal interpretation implies that the mass in the universe creates an inertial frame, and further conjectures consider that any asymmetric interaction with such an inertial frame would impart its reaction forces to the surrounding mass of the universe, thereby satisfying conservation of momentum. Although this notion has been raised as a theoretical issue for contemplating space drives (refs. 2, 16, and 17), it has not been fully explored. Any mechanical oscillation proposals invoking this approach would have to develop and subject a formalism for this concept to the normal rigor of a peer review. This should be a prerequisite to considering theoretical proposals along these lines.

Although there is theoretical and experimental work in the peer-reviewed literature that deals with Mach's principle and its propulsive implications, namely that of Jim Woodward's transient inertia effect (ref. 17), this transient inertia approach is not in the category of a mechanical oscillation thruster. At the time of this writing the theories and experimental findings regarding the transient inertia claims and their propulsive potential are still under investigation in the open literature and have not yet been independently confirmed or refuted.

\section{Gyroscopic Antigravity}

Another category of commonly purported mechanical breakthroughs consists of a system of gyroscopes. A famous example is from the 1973 demonstration by Eric Laithwaite, where a spinning gyro is shown to rise upward while it is forced to presses (ref. 18). Although such upward motion is a consequence of conservation of angular momenta, it is easily misinterpreted as an "antigravity" effect (ref. 19). Laithwaite, a Professor of Applied Electricity at the Royal Institution of Great Britain, 19671975 (ref. 20), went on to patent a device (fig. 7), that claims to produce linear force from such torques (ref. 21).

Variations on the theme of using gyroscopes that claim linear thrust are common and typically consist of forcing the axis of a spinning gyroscope to change its orientation in a manner that then causes the entire gyro to shift upward, thereby creating the impression that an upward "antigravity" force exists. Because a rigorous analysis of this dramatic motion can be difficult, the ambiguities regarding its real physics linger.

Such concepts can be viewed as trying to reverse the effect of a spinning top. Rather than falling immediately over, a spinning top precesses. It appears as if an invisible force is holding it up (fig. 8). Although the gravitational field, $g$, which is tilting the top, is linear, the way that the top reacts to it produces torques. These torques are working to conserve angular momentum in response to gravity. The notion of reversing this process, of forcing the gyroscopic precessions to induce a linear force opposing gravity ("antigravity"), seems like reasonable symmetry to expect, but this is not the case.

To illustrate the basic operation of a typical gyroscopic antigravity device, a simple version based on the Laithwaite demonstration (fig. 9) will be used at the working example. Laithwaite's demonstration consisted of just a single $50 \mathrm{lb}$ gyro rather than the dual version shown, but the principle of operation is the same. With the gyro up to speed, Laithwaite was able to lift the gyro by its stem (nonrotating beam that is coincident with the gyro's axis), by torquing the stem around horizontally. In figure 9, a dual version of the same thing occurs with the stems of two gyroscopes are mounted to a "main spindle." When this spindle is rotated, the gyros with their stems will pivot upward giving the impression of a lifting force. A mechanical stop is included to halt the gyro's tilting while it is still aligned to produce this "upward" force. 


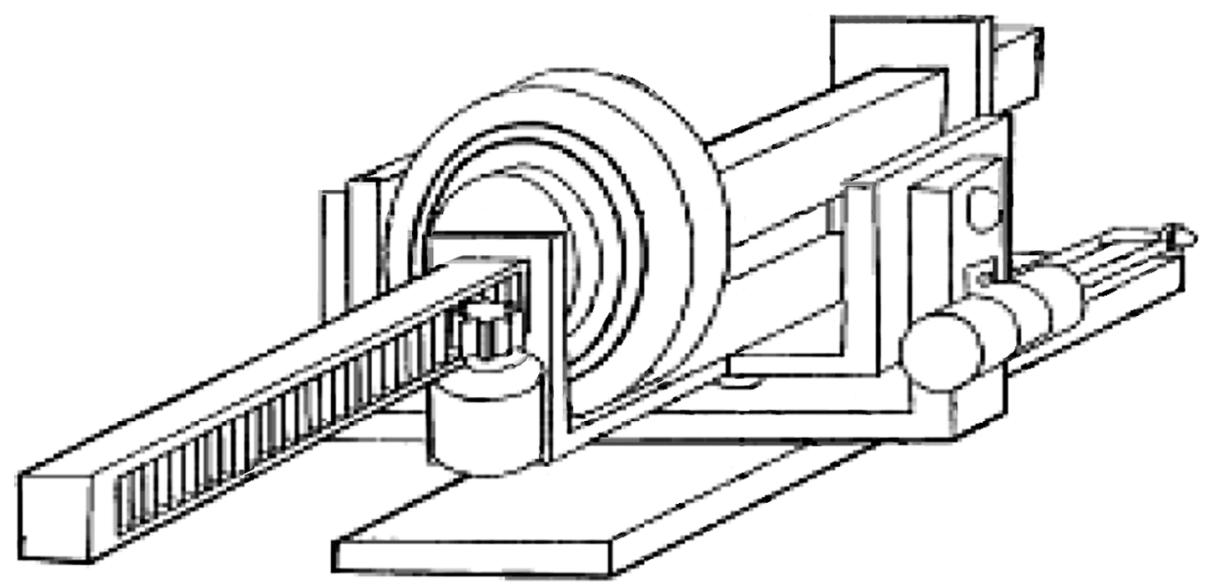

Figure 7.-Laithwaite: "Propulsion system". Patent quoting from US Patent $5,860,317:$ "... the mass of the first gyroscope moves with an associated second movement of the mass of the remainder of the system in substantially the opposite direction, wherein the movement owing to the translation-dominated portion and is larger than the movement owing to the precession-dominated portion of the motion, hence moving the system" (ref. 21).

Before

Top view

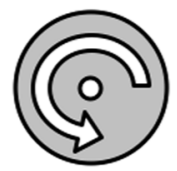

(1) The projection of ang' momentum of top reduced relative to vertical axis when tilted

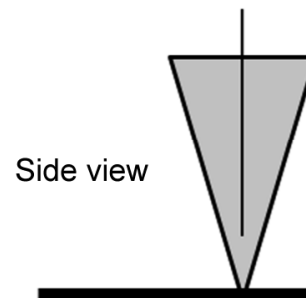

After

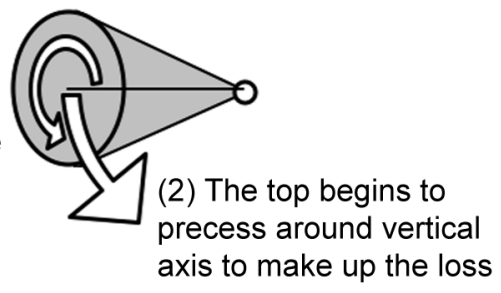

(2) The top begins to axis to make up the loss
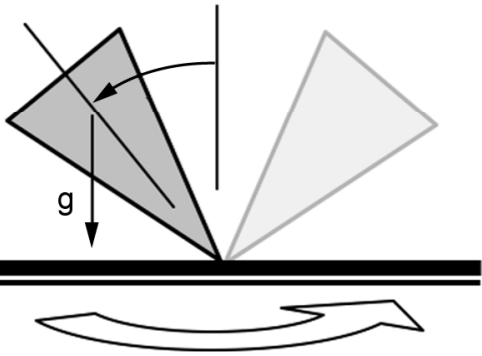

Figure 8.-Dynamics of a spinning top as gravity, g, pulls downward on a spinning top, the tilting changes the proportion of the top's angular momentum that is projected onto the vertical axis. To conserve angular momentum along this axis, the entire top will begin to presses around. This figure is intended as a mnemonic device to help understand the direction of such precessions rather than as a rigorous analysis of the system's dynamics. 


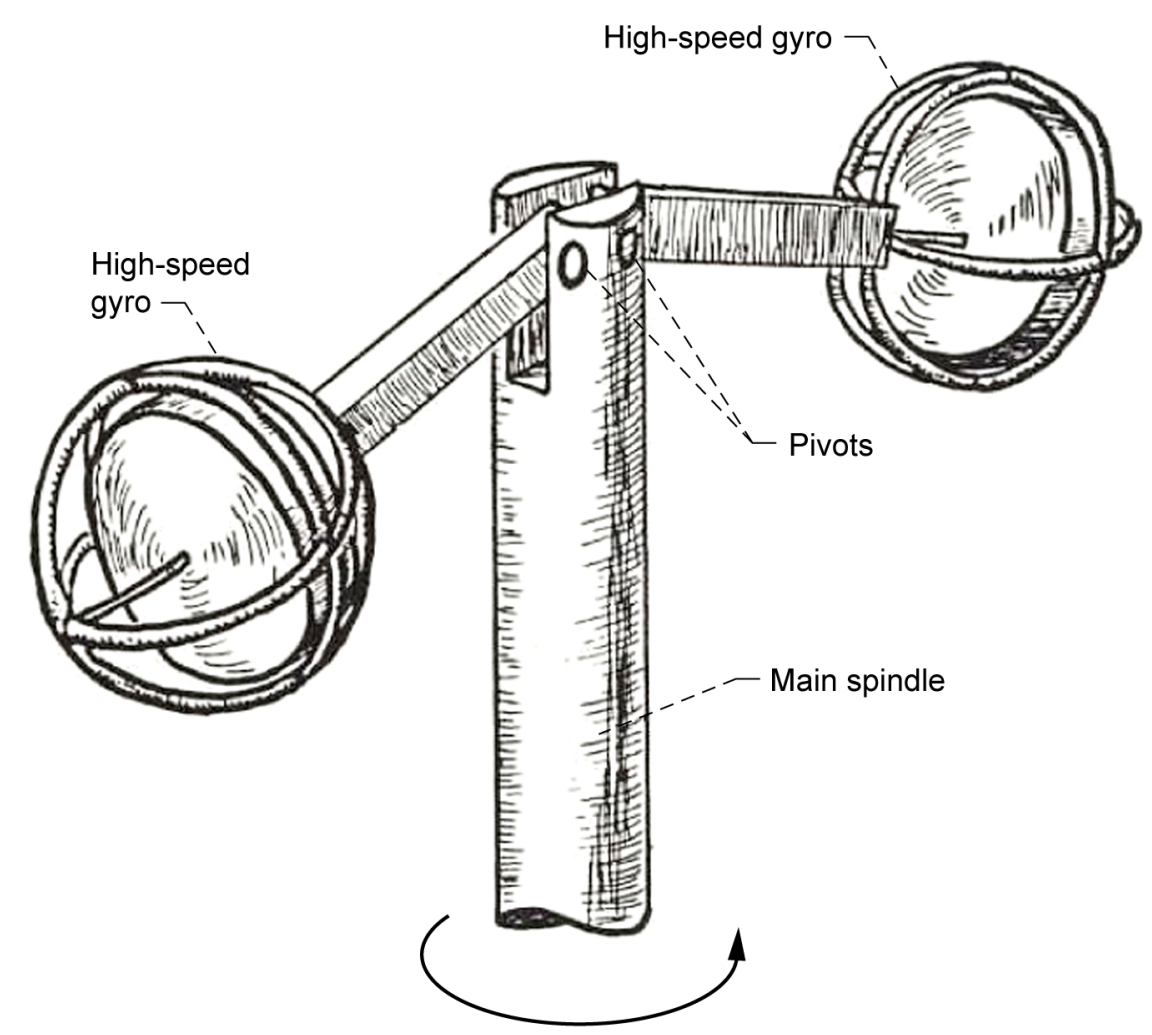

Figure 9.-Classical gyroscopic antigravity claim: "Essential design of the Laithwaite engine" (ref. 19). This is a typical version of a gyroscopic device that purports to exhibit an antigravity effect (taken from page 20 of Childress (ref. 19)). With the gyroscopes spinning, the main spindle is torqued. This causes the gyroscopes to flip upward at the pivot points, giving the impression that some antigravity effect is lifting them.

To understand this in terms of known physics, figure 10 presents a visual means to help understand how conservation of angular momenta can lead to such effects. Again, this diagram should only be interpreted as a mnemonic device for understanding the direction of torques involved with such mechanical devices, rather than as a rigorous means to fully convey the system's dynamics. Figure 10 shows the device before and after the main spindle has been torqued. A helpful perspective to comprehend how conservation of angular momentum functions is to only consider one axis at a time, so this illustration only concerns itself with the angular momentum in the axis of the main spindle. To set the initial angular momentum to zero, both the angular momentum of the main spindle and the gyroscope will be set to zero in this view. Therefore, the position of the gyro is set perpendicular to the main spindle so that it projects none of its angular momentum along the axis of the main spindle. Or said another way, when viewed from the top, there are no apparent rotations. Along the view of this axis, there is a zero angular momentum.

Next, in the after part of figure 10, the main spindle is rotating clockwise. (At this point it is not necessary to consider what affected this change, as this illustration is only intended to help visualize the torque directions from angular momenta conservation.) Since the rotation of the main spindle has introduced an angular momentum in this view, the stem of the gyro has shifted its alignment so that the gyro now presents a countering angular momentum so that their sum conserves the initial zero-valued angular momentum. When tilted, the projection of the gyro in this view now provides a rotational contribution. Even though it is only an ellipse in this view (circle viewed at an angle), this is enough to project a portion of its angular momentum into the plane of rotation of the spindle. When the gyros' stems 


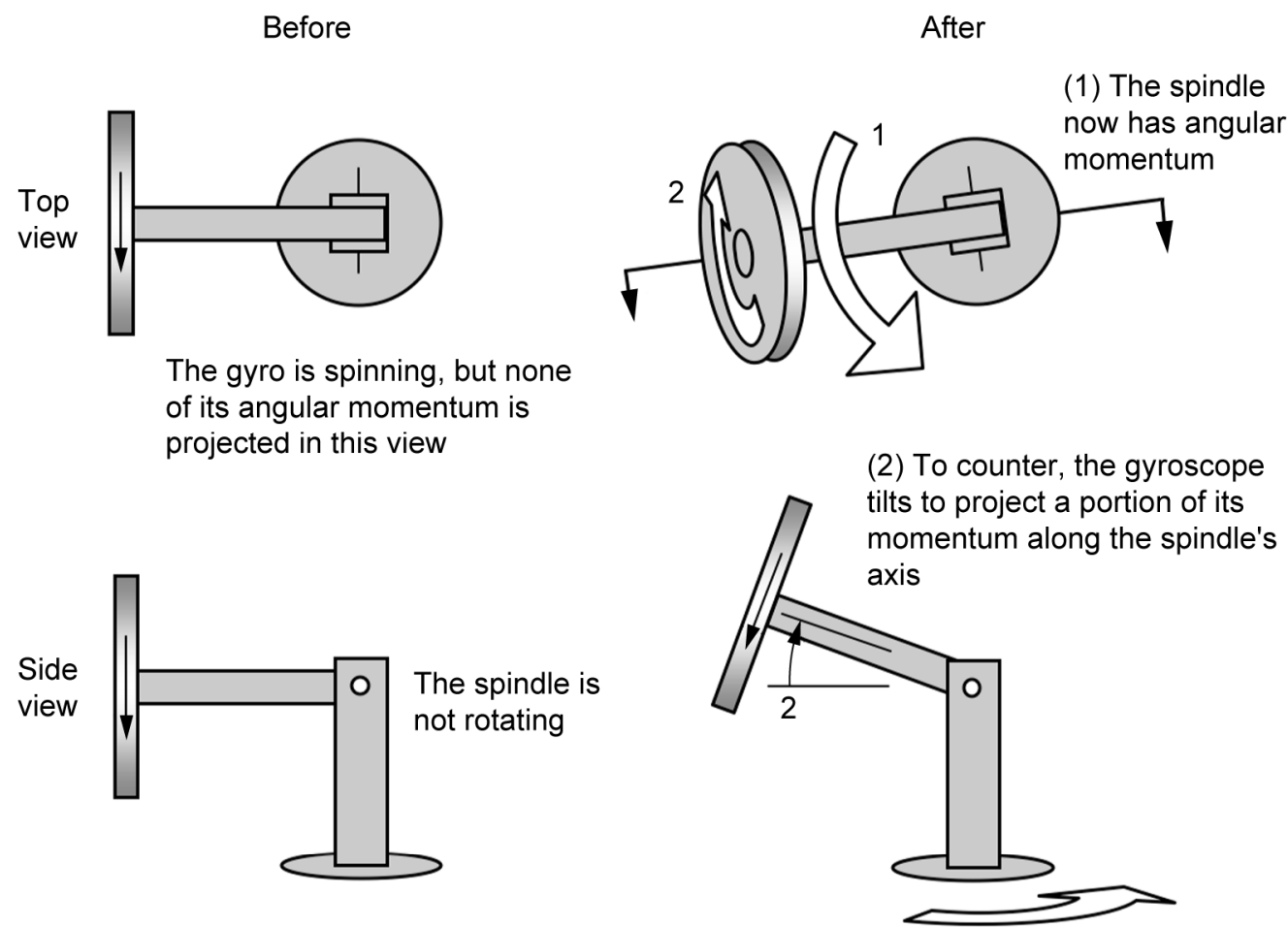

Figure 10.-Picturing conservation of angular momenta. The angular momentum of both situations is identical, but achieved differently. On the left, the gyroscope's rotation is aligned perpendicular to the axis of the spindle (zero contribution along the viewed axis) and the spindle is not rotating, hence the total angular momentum is zero. On the right, the spindle has been set into motion, introducing its own angular momentum. To conserve angular momentum, the gyroscope tilts to project an opposing portion of its angular momentum in the axis of the spindle, so that their sum remains zero. This diagram is not a strict representation of the dynamics of this system, but rather a mnemonic to help understand the directions that such coupled wheels will move.

reach the end of their allowed tilting angle, the torque that induced the tilt will still exist, but it will be acting between the pivot, stem, and the stem's stop. It is not an "upward" force relative to the gravitational field, but rather a torque.

Experimental testing of these gyroscopic devices is not as easy as with the oscillation thrusters. The key difference is that the gyroscopic thrusters require alignment with the Earth's gravitational field to operate. They do not produce lateral thrust that can be tested with the pendulum methods previously discussed. Instead, their weights must be measured. This is difficult because torques are introduced between the device and the platform on which it is mounted - reaction forces from its internal mechanisms. Furthermore, the frequency of vibrations from the gyroscopes might interfere with the operation of any weight-measuring device.

Drop tests, where fall times are measured to detect any changes in gravitational acceleration, are not likely to be a viable testing option for two reasons. Typical gyroscopic thrusting devices require the presence of the gravitational field, which vanishes in free-fall. To illustrate this with a simple experiment, drop a spinning top that is precessing. The moment that it begins its descent, the precessing will stop. The second reason that drop tests are not viable is because the device is not likely to survive the rapid deceleration at the end of its fall.

One testing option, as illustrated in figure 11, is to place two identical devices on each end of a balance beam and look for any tilting of the balance beam when one device is free to operate normally and the other has its stems locked to prevent the upward motion of the gyros. Even this simple test can be 


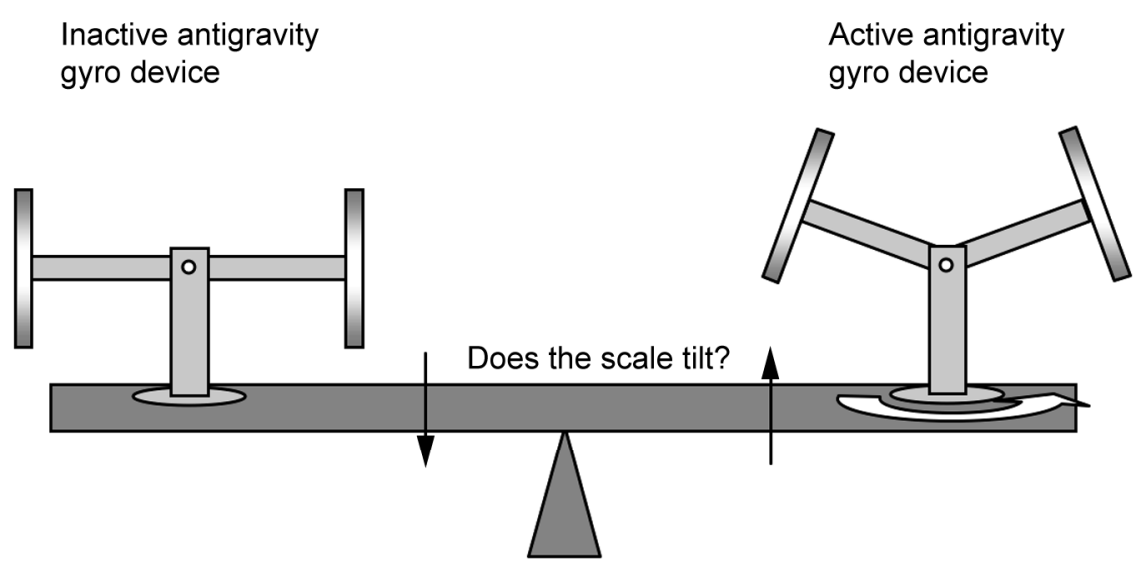

Figure 11.-Testing antigravity gyroscopes. One method of testing the antigravity claims of gyroscopic devices is to place two at either end of a balance beam and to see if there is any difference between an inactive and operating device. Even though an impulse is expected as the gyros begin their upward tilt, a countering impulse is equally expected when the gyros reach the end of their allowed travel and stop tilting upward. This sequence of impulse is likely to set the balance into oscillation, but a sustained tilting of the balance beam is not expected.

difficult because it requires two devices and can be vulnerable to spurious effects that could tilt a sensitive balance. Also, the transient impulses from the starting and stopping of the gyroscopes' tilting will induce oscillations in the balance beam.

To keep an open, yet rigorous, mind to the possibility that there has been some overlooked physical phenomena, it would be necessary that any future proposals on these types of devices explicitly address all the conventional objections, and provide convincing evidence to back up the claims. Any test results would have to be rigorous, impartial, and address all possible causes that might lead to a false-positive conclusion.

\section{Similar Concepts Not in This Category}

It is necessary to make clear distinctions between these gyroscopic antigravity devices and similarsounding devices, specifically: "reaction wheels," spinning superconductors, rotating masses in general relativity, anomalous right-hand rotation weight loss, and gyroscopic variants of the previously described oscillation thrusters.

A "reaction/momentum wheel" or "torque wheel" is an established device used in satellites to change the satellites' pointing direction (ref. 22). Even though the angular momentum of the entire system remains constant, the angular orientation and momentum of the external structure and its internal masses can be changed with respect to one another. For example, by rotating an internal wheel clockwise, the external cage (i.e., satellite body) will rotate counterclockwise. This is a simple and effective way to change the pointing direction of satellites, but such devices cannot be used to change the position of the center of mass of the system.

Another similar-sounding claim is the "gravity shielding" claim involving spinning superconductors (ref. 23). These superconductor claims were later shown not to be reproducible (ref. 24). Further claims of "Gravitomagnetic" effects using spinning superconductors (ref. 25) are still under review at the time of this writing. These are separate effects that should not be confused with claims of gyroscopic antigravity.

The concept of using rotating masses to induce forces does have a treatment within general relativity that can lead to acceleration fields, but it is through the very feeble effect of frame-dragging. In 1963, Robert Forward calculated the induced acceleration field from a rapidly rotating ultra-dense toroid 
(ref. 26). The magnitude of the induced effect is impractically trivial compared to the configurations needed to produce the effect. This example does serve, however, as a theoretical treatise on the subject.

In 1989 there were reports where a flywheel appeared to lose weight when rotating clockwise (when viewed from above) when its axis was aligned parallel to the earth's gravitational field. Oddly, no weight change was observed during counterclockwise rotation under otherwise identical circumstances (ref. 27). Two separate attempts to replicate these observations, using higher degrees of sensitivity, failed to confirm any such effect (refs. 28 and 29). Since this concept does not involve changing the position or orientation of the gyro's axis, it is not in this category of "gyroscopic antigravity"

When gyroscopic devices constrain their motions to a single plane, instead of the multi-direction axes versions that are the focus of this section, then they are just a variation of the oscillation thrusters discussed previously. The version shown in figure 2 is a classic example.

\section{Preparing Responses}

Since amateurs are most often the ones who submit mechanical antigravity proposals, professional assessment methods such as journal peer-reviews or analytical assessments are ineffective. Most amateurs are not able to follow such processes. Furthermore, amateur correspondence can be emotionally charged, adding to the difficulty of preparing a helpful response. This section provides suggestions for addressing these non-technical aspects of responding to unsolicited proposals.

From the statistics collected with the Breakthrough Propulsion Physics Project shown in table I, roughly a third of the amateur correspondences (specifically 9 percent of all unsolicited correspondence), displayed delusions of grandeur or paranoia. Based on the advice of a psychologist, it is recommended to not send any response at all (Author's discussion with Dr. Joseph R. Wasdovich, Employee Assistance Program Manger and psychologist, NASA Glenn Research Center, Dec. 11, 2002). The reason is that a technical response will not provide the submitter with the kind of help they need and will only encourage more unproductive correspondence. Delusions of grandeur are evidenced when the submitter states that their device or theory is the answer to solve the worlds problems, or other statements to that effect. Paranoia is easier to recognize, where the submitter expresses fear about their safety. Often these characteristics coexist, such as when the submitters express concern that their submission is so valuable that it will evoke suppression by evil conspirators.

Most of the amateur submissions, however, are curiosity-driven and the amateurs simply do not know where to turn for guidance. As previously stated, normal venues for assessments are excessively difficult for amateurs, so it has been found to be more effective to give the submitters a task that is within their ability to perform and that will educate them to the critical details. Sometimes this can be as simple as showing them an example of a similar, previously submitted, idea that was proven not to work as claimed. The examples presented in the figures of this report might serve this purpose depending on the nature of the device offered.

In cases where the submitters have built devices, it is recommended that the response dictate further experimental tests as a condition of deeper inquiry. This includes recommending the level pendulum test for oscillation thrusters (fig. 4) as well as outlining known causes of false positives, such as those illustrated in figures 5 and 6 . By giving the submitter a reasonable next step and advice on what pitfalls to avoid, it gives them the means to learn from their own experiences. By making successful tests a condition for engaging in future correspondence, it relieves reviewers from further time-consuming correspondence, yet leaves open the option of learning about possible positive results.

When drafting the response letter, it is not effective to simply dismiss the ideas as violating known physics. This tends to evoke inflammatory, emotionally charged correspondence, plus it does not give the submitters a path to convincing themselves of the real operation of their device. It is necessary in the response to raise the issue of a violation of known physics, but in a way that leaves them an option to prove otherwise. For example, the following is an example of a more effective response: 


\begin{abstract}
"Your device appears to violate conservation of momentum, a well-evidenced law of nature. To convince us that something other than this is occurring with your device, we require that you perform additional tests to a higher standard of proof. More detailed suggestions are included for such tests as well as explanations for why other devices, that appear similar to yours, were found not to be viable propulsion devices. If after following our suggestions and ensuring that all false-positive effects have been dismissed and you then have convincing evidence that your device is operating in an effective and novel manner, we will gladly reconsider your submission. Until such conditions are met, we regret that we do not have the resources to maintain correspondence or provide further assistance in your investigations. We wish you the best in your endeavors."
\end{abstract}

This response puts the burden of proof back on the submitter, setting clear and reasonable steps to move ahead to the next level of legitimacy.

\title{
Conclusion
}

Unsolicited submissions of claimed breakthroughs that are based on errant interpretations of mechanical forces are common. Two devices in particular involve oscillating masses that claim net thrust and gyroscopic devices that claim antigravity effects. The oscillation thrusters are misinterpretations of differential friction, while the gyroscopic devices misinterpret torques as linear thrust. To help reduce the burden on reviewers and to give would-be submitters the tools to assess these ideas on their own, examples of these devices, their operating principles, and testing criteria are offered. By putting the burden of proof on the submitter and using these examples to help, the submitter has a better chance of learning how their devices truly operate.

\section{References}

1. Millis, Marc, G., 2004, Breakthrough Propulsion Physics Project: Project Management Methods, NASA/TM-2004-213406. Available at: http://gltrs.grc.nasa.gov/citations/all/tm-2004-213406.html

2. Millis, Marc, G., 2005, Assessing Potential Propulsion Breakthroughs. In New Trends in Astrodynamics and Applications. ed. Edward Belbruno. New York: Annals of the New York Academy of Sciences, 1065: pp. 441-461.

3. Rogers, Adam, 1998, "Department of Warp Drive and Wormholes," Newsweek, (31 August): 12.

4. Greenwald, J., 1998, "To Infinity... and Beyond: Warp drive, wormholes, and the power of nothing," Wired (July): pp. 90-97.

5. DiChristina, Mariette, 2001, "Space at Warp Speed: Wormholes, negative energy, and Star Trek-like space drives are the stuff of real research at NASA," Popular Science (May): pp. 46-51.

6. Mallon, Thomas, 2003, "Onward and Outward," The New York Times, (5 February): Editorial/Op-Ed.

7. Daniels, Patricia, 2002, "Actually, it does take a rocket scientist: Amateurs launch slew of ideas for space travel," The Boston Globe (12 November): C1.

8. Gilster, Paul, 2004, Centauri Dreams: Imagining and Planning Interstellar Exploration. New York: Copernicus Books.

9. Shatner, William, and Chip Walter, 2002, I'm Working On That. Simon and Schuster.

10. Dean, Norman L., 1959, "System for Converting Rotary Motion into Unidirectional Motion," US Patent 2,886,976 (Filed Jul. 13, 1954, granted May 19, 1959).

11. Foster, Sr., Richard E., 1997, "Inertial Propulsion Plus/Device and Engine," US Patent 5,685,196 (Filed July 16, 1996, Granted Nov. 11, 1997).

12. Thornson, Brandson R., 1986, “Apparatus for Developing a Propulsion Force,” US Patent 4,631,971 (Filed May 25, 1984, Granted Dec. 30, 1986).

13. Resnick, Robert, and David Halliday, 1977, Physics (3rd ed). John Wiley \& Sons, NY: 123, 314.

14. Davis, 1962, "The Fourth Law of Motion," Analog Science Fiction, Science Fact, (May): pp. 83-105. 
15. Mach, E., 1883, The Science of Mechanics, Fifth English Edition, Open Court Publishing Co., London, (1942).

16. Millis, Marc, G., 1997, "Challenge to Create the Space Drive," AIAA Journal of Propulsion and Power, 13(5): pp. 577-582.

17. Woodward, James F., 2004, "Flux Capacitors and the Origin of Inertia," Foundations of Physics, $34: 1475-1514$.

18. Eigenbrot, Ilya V., 2004, "Re: Error on website," Email exchange between Dr. Ilya V Eigenbrot, International and Science Communication Officer, Imperial College London, and Marc Millis, NASA Glenn Research Center, Cleveland (15 Sept 2004).

19. Childress, D. Hatcher, 1985, The Anti-Gravity Handbook, Adventures Unlimited Press, Stelle Illinois: $18,20$.

20. The Royal Institution- Heritage: Ri People. 2006. Royal Institution of Great Britain, London UK. http://www.rigb.org/rimain/heritage/ripeople.jsp (Accessed 1 June 2006).

21. Laithwaite, Eric and William Dawson, 1999, "Propulsion System," US Patent 5,860,317 (Filed 5 Nov. 1996, Granted 19 Jan. 1999)

22. Bayard, David S., 2001, An Optimization Result With Application to Optimal Spacecraft Reaction Wheel Orientation Design, Proceedings of the American Control Conference 2001, Arlington, VA June 25-27, 2001: pp. 1473-1478, Vol. 2.

23. Podkletnov E., and Nieminen, 1992, A Possibility of Gravitational Force Shielding by Bulk YBCO Superconductor. Physica C, 203: pp. 441-444.

24. Hathaway, George., B. Cleveland, and Y. Bao, 2003, Gravity modification experiment using a rotating superconducting disk and radio frequency fields. Physica C, 385: pp. 488-500.

25. Tajmar, Martin. F., Plesescu, K. Marhold, and Clovis J. de Matos, 2006a, Experimental Detection of the Gravitomagnetic London Moment. Submitted to Physica C. http://arxiv.org/abs/gr-qc/0603033 (Accessed 13 Mar. 2006).

26. Forward, Robert, 1963, Guidelines to Antigravity. American Journal of Physics, 31: pp. 166-170.

27. Hayasaka, Hideo and Sakae Takeuchi, 1989, Anomalous weight reduction on a gyroscope's right rotations around the vertical axis on the Earth. Phys. Rev. Lett. 63: pp. 2701-2704.

28. Faller, J.E., W.J. Hollander, P.G. Nelson, and M.P. McHugh, 1990, Gyroscope-weighing experiment with a null result. Phys. Rev. Lett. 64: pp. 825-826.

29. Nitschke, J.M. and P.A. Wilmarth, 1990, Null result for the weight change of a spinning gyroscope. Phys. Rev. Lett. 64: pp. 2115-2116. 


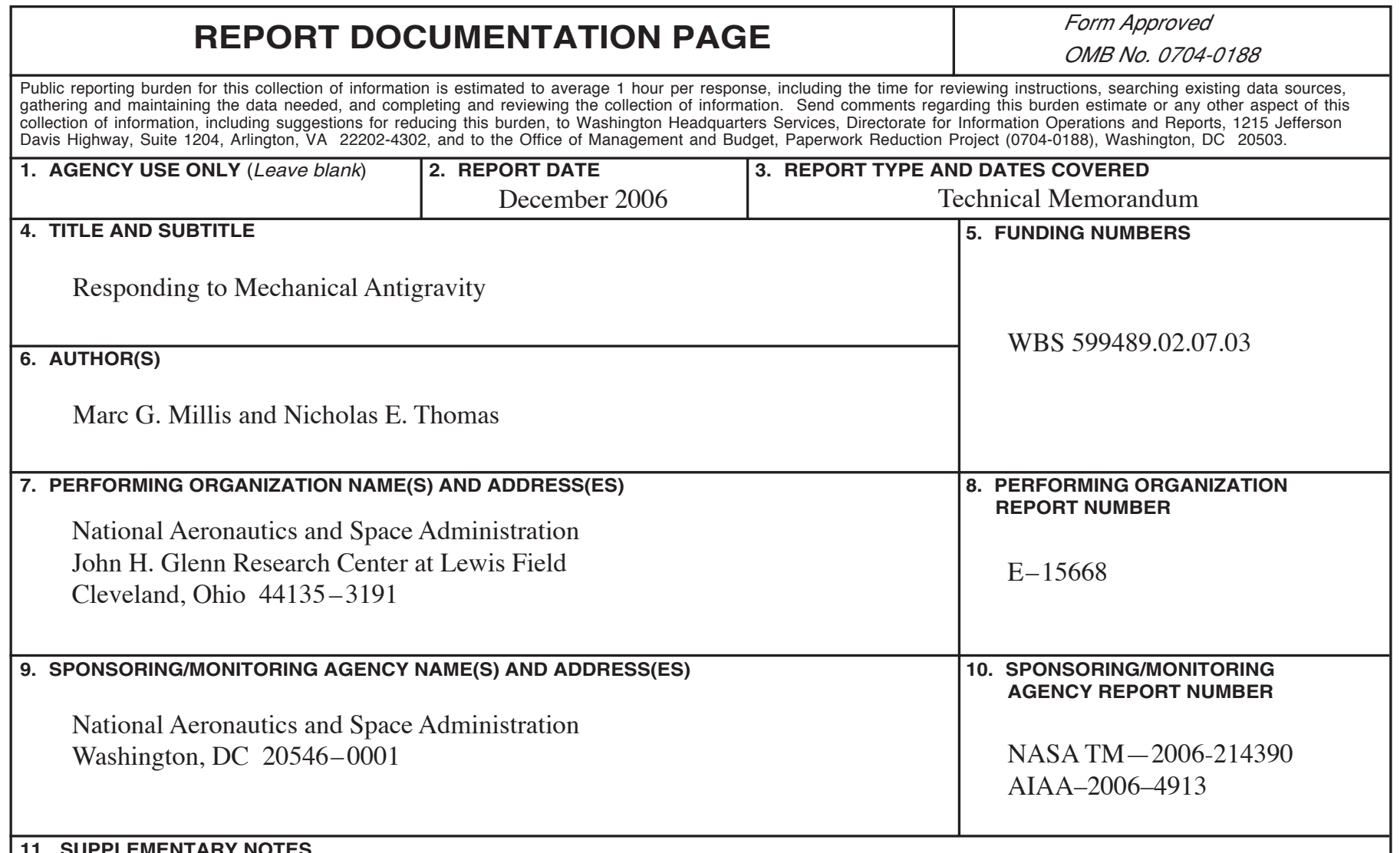

11. SUPPLEMENTARY NOTES

Prepared for the 42nd Joint Propulsion Conference and Exhibit, cosponsored by the AIAA, ASME, SAE, and ASEE, Sacramento, California, July 9-12, 2006. Marc G. Millis, NASA Glenn Research Center; and Nicholas E. Thomas, University of Miami, P.O. Box 248106, Miami, Florida 33124. Responsible person, Marc G. Millis, organization code RTP, 216-977-7535.

\begin{tabular}{|l|l|l|l|}
\hline 12a. DISTRIBUTION/AVAILABILITY STATEMENT & 12b. DISTRIBUTION CODE
\end{tabular}

Unclassified - Unlimited

Subject Categories: 12, 20, and 70

Available electronically at http://gltrs.grc.nasa.gov

This publication is available from the NASA Center for AeroSpace Information, 301-621-0390.

13. ABSTRACT (Maximum 200 words)

Based on the experiences of the NASA Breakthrough Propulsion Physics Project, suggestions are offered for constructively responding to proposals that purport breakthrough propulsion using mechanical devices. Because of the relatively large number of unsolicited submissions received (about 1 per workday) and because many of these involve similar concepts, this report is offered to help the would-be submitters make genuine progress as well as to help reviewers respond to such submissions. Devices that use oscillating masses or gyroscope falsely appear to create net thrust through differential friction or by misinterpreting torques as linear forces. To cover both the possibility of an errant claim and a genuine discovery, reviews should require that submitters meet minimal thresholds of proof before engaging in further correspondence; such as achieving sustained deflection of a level-platform pendulum in the case of mechanical thrusters.

14. SUBJECT TERMS

Spacecraft propulsion; Antigravity; Mechanical oscillators; Gyroscopes

\begin{tabular}{|c|c|c|c|}
\hline $\begin{array}{c}\text { 17. SECURITY CLASSIFICATION } \\
\text { OF REPORT } \\
\text { Unclassified }\end{array}$ & $\begin{array}{c}\text { 18. SECURITY CLASSIFICATION } \\
\text { OF THIS PAGE } \\
\text { Unclassified }\end{array}$ & $\begin{array}{c}\text { 19. SECURITY CLASSIFICATION } \\
\text { OF ABSTRACT } \\
\text { Unclassified }\end{array}$ \\
\hline
\end{tabular}

NSN 7540-01-280-5500

Standard Form 298 (Rev. 2-89) 

